\title{
Experimental Investigation of Performance of the Wind Turbine with the Flanged-Diffuser Shroud in Sinusoidally Oscillating and Fluctuating Velocity Flows
}

\author{
Kazuhiko Toshimitsu ${ }^{1}$, Hironori Kikugawa ${ }^{1}$, Kohei Sato ${ }^{2}$, Takuya Sato ${ }^{1}$ \\ ${ }^{1}$ Department of Mechanical Engineering, Oita National College of Technology, Oita, Japan \\ ${ }^{2}$ Department of Mechanical Engineering, Kyushu University, Fukuoka, Japan \\ Email: tosimitu@oita-ct.ac.jp
}

Received September 9, 2012; revised October 15, 2012; accepted October 26, 2012

\begin{abstract}
The wind turbine with a flanged-diffuser shroud - so called "wind-lens turbine"-is developed as one of high performance wind turbines by Ohya et al. In this paper, the wind turbine performance is investigated for both steady and unsteady winds. The compact-type wind lens turbine shows higher efficiency than the only rotor wind turbine. Also, the flow structure around the compact-type wind turbine is made clear by CFD and PIV in steady wind. Furthermore, the performances of the only rotor and the compact-type wind-lens turbines for unsteady wind are experimentally and numerically investigated. Experimental and numerical results are presented to demonstrate the dependence of frequency of the harmonic oscillating velocity wind on power coefficient. Consequently, the compact-type wind-lens turbine show better performance than the only rotor one in sinusoidally oscillating velocity wind. Furthermore, the numerical estimation can predict the power coefficient in the oscillating flows to an accuracy of $94 \%$ to $102 \%$. In addition, the dependence of the turbine performance on turbulent intensity and vortex scale of natural fluctuating wind is presented.
\end{abstract}

Keywords: Wind Turbine; Unsteady Flow; Wind Energy; Power Coefficients; Flanged Diffuser; Natural Wind

\section{Introduction}

It is important issue that a wind power generation is improved to raise the efficiency as one of natural energy sources in order to promote the usage of sustainable energy. According to the background, the wind turbine with a flanged-diffuser shroud - so called "wind-lens turbine" -is developed as one of high performance wind turbines by Ohya et al. [1]. In particular, the wind-lens turbine can generate electric power even in low velocity wind since the flanged-diffuser shroud increases the wind velocity at rotor. For the long and compact type wind lens turbines, we studied the detail flow structure around the wind turbine with a particle image velocimetry in authors' previous work [2]. Recently, research subjects of wind turbines in unsteady flows become important, which are the effects of fluctuating wind velocity and flow direction, non-uniform inflow, turbulence and other factors. They influence the performance and fatigue problem. In particular, wind in Japan is more unstable than western country, namely wind velocity and flow direction are easy to fluctuate. Thus it is important that the characteristics of the turbines in unsteady wind should be made clear. In previous concerned works, the numerical performance estimation of the oscillating wind velocity is proposed by Karasudani et al. [3-5].

In this paper, we will focus on the effect of the fluctuating wind velocity upon the wind turbine performance. The performances of the ordinary rotor wind turbine and the compact-type wind-lens turbine in harmonic oscillating velocity wind are experimentally and numerically investigated. In particular, experimental and numerical results are presented to demonstrate the dependence of the wind velocity frequency on power coefficient of the wind turbines. Furthermore, the effects of the turbulent intensity and vortex scale in natural fluctuating velocity wind on the turbines performance are studied.

\section{Experimental Apparatus}

\subsection{Wind Turbine Model}

The schematic designs and photographs of the compacttype wind-lens turbine are shown in Figure 1. The optimum diffuser profile and the flange height are determined by Ohya et al. [1]. The rotors are designed for the two tip-speed ratios, $\lambda_{D}=3.7$ and 5.0. Here the basic blade profile of $\lambda_{D}=5.0$ is designed by Furukawa et al. [6]. The blade cross section profile is changed from 
NACA63218 at root to NACA63212 at tip along a span. The specific dimensions of the diffusers and the rotor are listed in Tables 1 and 2 respectively.

\subsection{Measurement System of the Wind Turbine Performance}

The torque and rotational speed of the rotor are measured by the experimental apparatus as shown in Figure 2. The wind turbines are set up at the center of cross section of the wind tunnel. The wind turbine power measurement system is consisted of the torque detector, the rotational speed sensor, the torque converter, the DC motor and the DC power supply. The DC motor and the DC power supply work as a power generator, which control the rotor load and the rotational speed. Here the generated air flow is actively controlled by the 66 fans of the multi-fan wind tunnel in ONCT.

\section{Wind Turbines Performance in Steady Flow}

\subsection{Power Coefficients of the Wind Turbines in Steady Flow}

The power coefficients of wind turbine are defined by

$$
C_{w}=\frac{P}{\rho A U^{3} / 2},
$$

where $P, \rho, A$ and $A$ denote generated power of a wind turbine, fluid density, wind receiving area and the upstream wind velocity, respectively. The power coefficients of the only rotor and the compact-type wind turbines are normalized by the rotor swept area $A=\pi D_{r}^{2} / 4=$ $2.96 \times 10^{2} \mathrm{~m}^{2}$ and the flange circular area $A=\pi D_{r}^{2} / 4=$ $5.56 \times 10^{2} \mathrm{~m}^{2}$, respectively. It is found that the maximum power coefficients $C_{w}$ of the compact-type wind turbines are 1.5 times as large as the only rotor in Figure 3. It means that the wind-lens turbine clearly shows higher efficiency than the conventional wind turbine.

\subsection{Flow Structures around the Wind-Lens Turbines in Steady Flow}

The importance of the flow structures behind the windlens turbines was pointed out to increase the velocity at rotor in the previous papers $[1,2]$. In order to investigate the mechanism of the increasing flow velocity at the rotor,
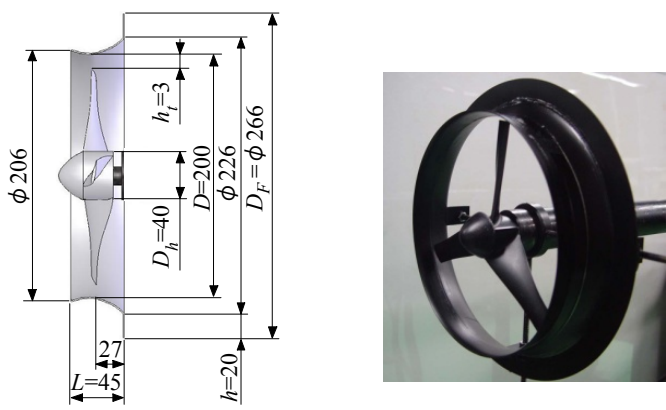

Figure 1. Schematics and photograph of the compact-type wind turbine with the flanged-diffuser shroud.

Table 1. Dimensions of the wind turbine with the flangeddiffuser shroud.

\begin{tabular}{|c|c|}
\hline Tip clearance, $h_{t}$ & $3 \mathrm{~mm}\left(h_{t} / D=1.5 \%\right)$ \\
\hline Diffuser length, $L / D$ & 0.225 \\
\hline Flange height, $h / D$ & 0.1 \\
\hline
\end{tabular}

Table 2. Dimensions of the rotor blades.

\begin{tabular}{ccc}
\hline Rotor diameter & $D_{r}(=2 r)$ & $194 \mathrm{~mm}$ \\
Hub to tip ratio & $D_{h} / D$ & 0.2 \\
Design tip-speed ratio & $\lambda_{D}=\omega r / U$ & $3.7,5.0$ \\
Blade profiles & NACA63218 (root) - NACA63212 (tip) \\
\hline
\end{tabular}

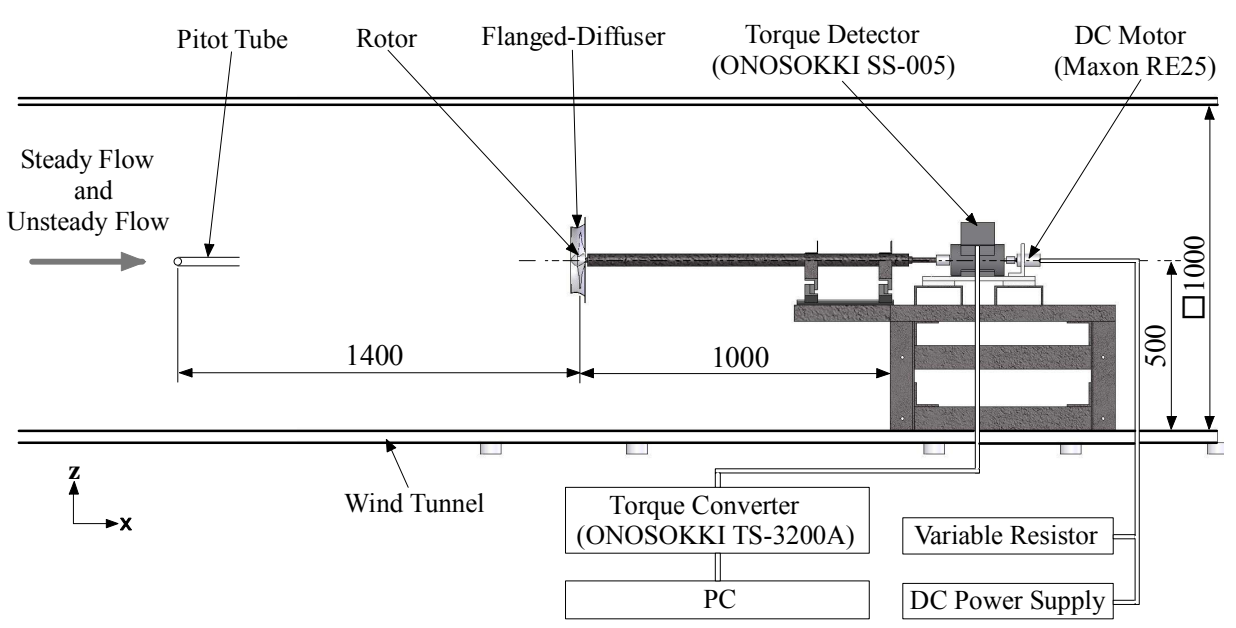

Figure 2. Schematics of measuring system of wind turbine performance. 
herewith we present an experimental and numerical timeaverage streamlines around the compact-type wind turbine at the tip-speed ratio $\lambda=3.9$ and mean velocity $\bar{U}=7 \mathrm{~m} / \mathrm{s}$. The experimental streamline by PIV measurement is shown in Figure 4. Also the CFD analysis with the commercial software ANSYS CFX 12 is presented in Figure 5. The numerical analysis is based on the 2nd order upwind implicit scheme for the conservation equation and $k-\varepsilon$ turbulence model. Figure 6 shows the multi-block 3-D mesh for the numerical analysis, which is consisted of 6.4 million nodes. The rotor mesh block rotates at $2700 \mathrm{rpm}$. The boundary conditions are identified as the inflow conditions of $\bar{U}=7 \mathrm{~m} / \mathrm{s}$, the exit pressure of $101.3 \mathrm{kPa}$, slip flows on the outside walls and nonslip flows on the rigid object surfaces.

The vortex structure behind the flange of both PIV and CFD results qualitatively agree with. Twin vortices are found behind the flange. According to the results, the flow structure is illustrated in Figure 7. The vortex " $A$ " behind the flange mainly caused the low pressure region. Thus it increases the wind velocity at rotor. The maximum velocity at rotor surface is calculated $9.5 \mathrm{~m} / \mathrm{s}$ as velocity rising.

\section{Performance in Sinusoidally Oscillating Velocity Flow}

In this chapter, we experimentally and numerically discuss the performance of the wind turbine in sinusoidally oscillating velocity wind.

\subsection{Experimental Conditions of Sinusoidally Oscillating Flow to Investigate the Wind Turbine Performance}

The upstream wind velocity of the wind turbine is undergoing identical harmonic oscillation as follow,

$$
U(t)=\bar{U}+\tilde{u} \sin (2 \pi f t) .
$$

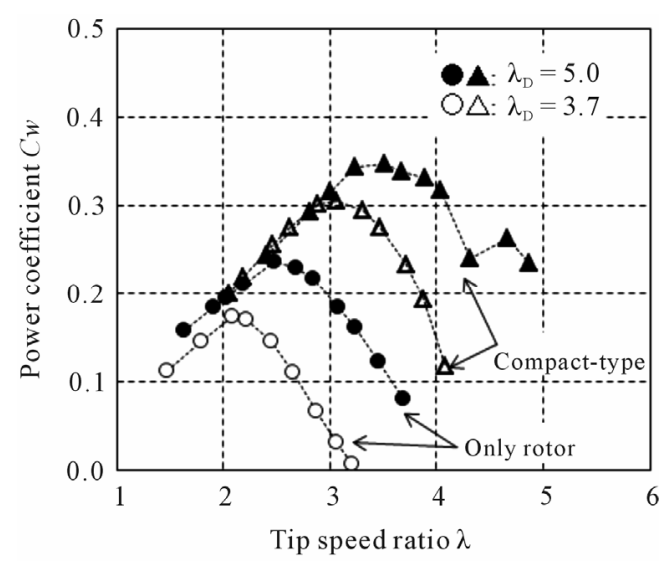

Figure 3. Relationship of power coefficients and tip-speed ratio in the steady wind velocity $5 \mathrm{~m} / \mathrm{s}$.

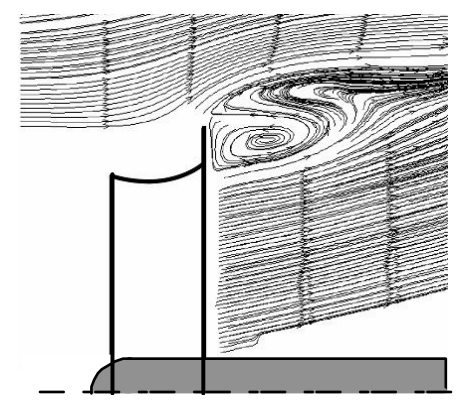

Figure 4. Time average meridional streamlines by PIV.

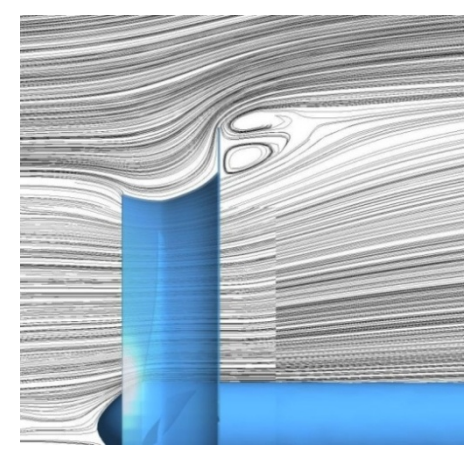

Figure 5. Time average meridional streamlines by the present CFD.

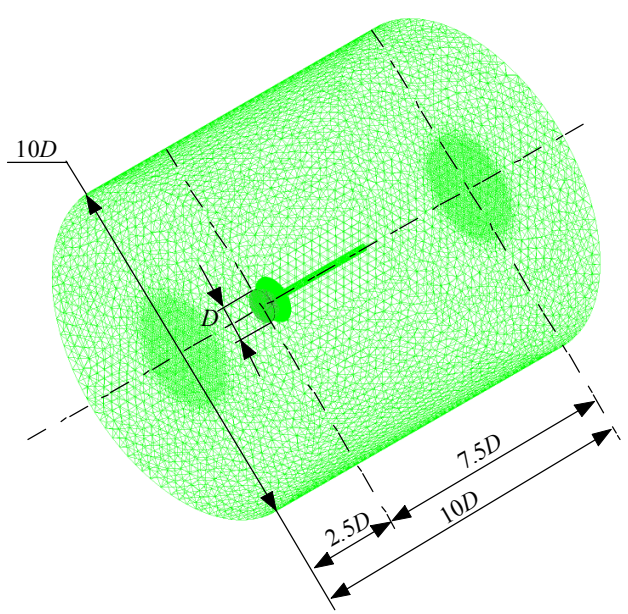

Figure 6. Multi-blocks unstructured mesh for calculation.

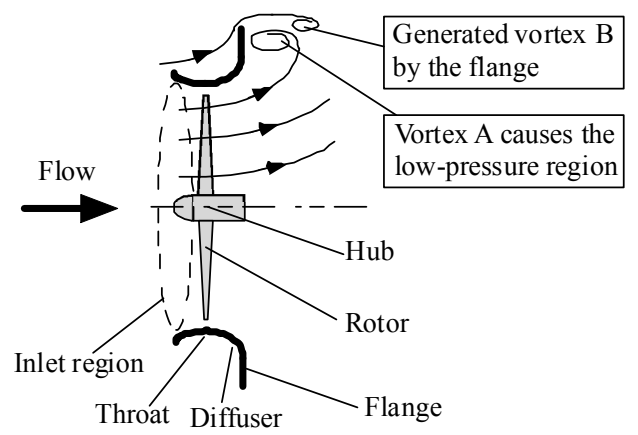

Figure 7. Schematic flow structures around the wind-lens turbine in steady wind. 
The performances of the wind turbines are investigated as the upstream mean velocities $\bar{U}=5 \mathrm{~m} / \mathrm{s}$ with oscillating amplitudes $\tilde{u}=1.0 \mathrm{~m} / \mathrm{s}$. The wind velocity oscillates at frequencies $f=0.033,0.05,0.083$ and $0.25 \mathrm{~Hz}$. Detail data are listed in Table 3. The time histories of the oscillating wind velocities at $\bar{U}=5 \mathrm{~m} / \mathrm{s}$ and $\tilde{u}=1.0$ $\mathrm{m} / \mathrm{s}$ with $f=0.25$ and $0.033 \mathrm{~Hz}$ are shown in Figure 8, which are measured at $1.4 \mathrm{~m}$ upstream location from the wind turbine front by a L-type Pitot tube. It is found that the experimental wind correctly oscillates with the specific conditions.

The characteristic frequency of the oscillating wind based on the turbine rotor dynamic response and the power coefficient in steady wind is proposed by Karasudani et al. [4] as follow,

$$
f_{r}=\frac{3 \rho \pi r^{4} C_{w}\left(\lambda_{w}\right) \bar{U}}{2 I \lambda_{w}^{2}},
$$

where $\rho, r, I, \lambda_{w}$ and $C_{w}\left(\lambda_{w}\right)$ mean fluid density, rotor radius, moment of rotor inertia, the tip-speed ratio at the maximum power coefficient and the estimated power coefficient integrated the power coefficients in the steady flow, respectively. (The details of the numerical treatment are described in the later Section 4.3.) The moment of inertia of the rotor is $I=1.52 \times 10^{-4}$ $\mathrm{kgm}^{2}$. Equation (3) has the basic assumptions which the

Table 3. Conditions of oscillating winds and turbine characters.

\begin{tabular}{|c|c|c|c|c|c|}
\hline $\begin{array}{c}\bar{U} \\
{[\mathrm{~m} / \mathrm{s}]}\end{array}$ & $\begin{array}{l}\tilde{u} \quad[\mathrm{~m} / \mathrm{s}] \\
(\tilde{u} / \bar{U} \%)\end{array}$ & \multicolumn{4}{|c|}{$\begin{array}{l}\text { Oscillation Frequency, } f[\mathrm{~Hz}] \\
\text { (Oscillation Period, } T=1 / f[\mathrm{~s}] \text { ) }\end{array}$} \\
\hline \multirow[t]{3}{*}{5.0} & $1.0(20 \%)$ & $0.25(4)$ & $0.083(12)$ & $0.05(20)$ & $0.033(30)$ \\
\hline & & \multicolumn{2}{|c|}{$\lambda_{w}$} & \multicolumn{2}{|c|}{$f_{r}\left(1 / 2 f_{r}\right)$} \\
\hline & $\lambda_{D}$ & 3.7 & 5.0 & 3.7 & 5.0 \\
\hline \multirow{2}{*}{$\begin{array}{l}\text { Wind } \\
\text { turbines }\end{array}$} & Only Rotor & 2.09 & 2.47 & $0.65(0.22)$ & $0.64(0.21)$ \\
\hline & Compact & 3.06 & 3.51 & $1.01(0.34)$ & $0.87(0.29)$ \\
\hline
\end{tabular}

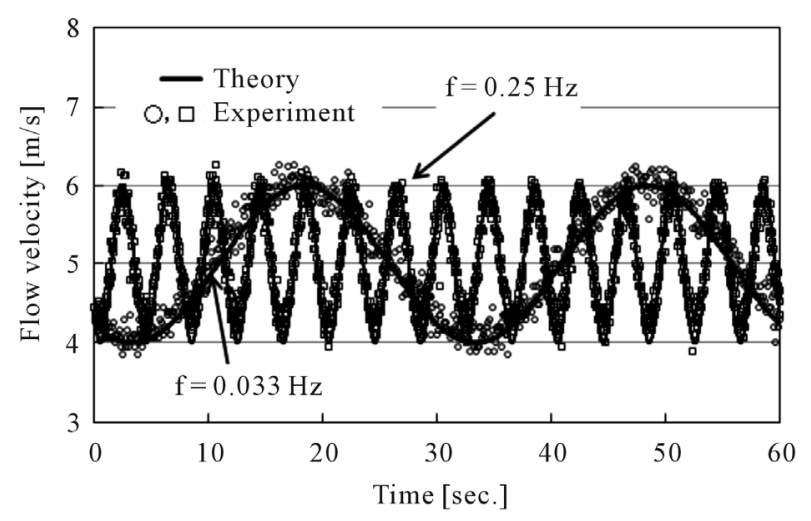

Figure 8. Comparison of experimental and theoretical oscillating wind velocities in the wind tunnel. rotor response is the quasi-steady state and turbine rotor torque is depended only on tip-speed ratio [3,4]. In other words, the flow transient time which the flow field around the rotor corresponds with the oscillation wind is sufficiently small, thus the quasi-steady state is satisfied. It means that the turbine rotor can responsively rotates to the fluctuating wind velocity for $f<f_{r}$. Here, we should mention that Karasudani et al. insist that the real only rotor turbine does respond for $f<f_{r} / 3$ by numerical simulation [4].

We are going to examine whether the quasi-steady response can be applied to this equipment. Let the rotor speed be $3000 \mathrm{rpm}$, i.e. the time of one rotation be $t_{r}=0.02$ seconds. If the inflow velocity decreases to 1 $\mathrm{m} / \mathrm{s}$, the flow pass at the rotor tip of the thickness $2 \mathrm{~mm}$ for $t_{b p}=2 \times 10^{-3}$ seconds. Since the time $t_{b p}$ is sufficiently smaller than $t_{r}$, thus the flow field around the rotor can enough changed during one rotation. Furthermore the rotor blade passing time $t_{r p}$ of the wind should be sufficiently smaller than the wind oscillating period. Since the axial length of the rotor equals about $4 \mathrm{~cm}$, the rotor blade passing time $t_{r p}$ equals 0.04 seconds. Hence $t_{r p}$ is much smaller than the wind oscillating period (4 seconds at $f=0.25 \mathrm{~Hz}$ ). Consequently, the flows around the rotor can be regarded as the quasi-steady state for this experiment. The experimental values $\lambda_{w}$ and $f_{r}$ are shown in Table 3.

\subsection{Power Coefficients of the Wind Turbines in Oscillating Velocity Flow}

In this section, we present the example results of the mean flow $\bar{U}=5 \mathrm{~m} / \mathrm{s}$ and $\tilde{u}=1.0 \mathrm{~m} / \mathrm{s}$ (i.e. $\tilde{u} / \bar{U}=$ $20 \%$ ) in the conditions of Table 3 . The effects of the wind frequencies $f$ upon the power coefficients $C_{w}$ as the function of the mean tip-speed ratio $\lambda$ for the only rotor and compact-type wind turbines with the designed rotor tip speed ratio $\lambda_{D}=5.0$ are shown in Figures 9 and 10.

The maximum coefficients are presented at $\lambda \cong 2.7$ and 3.5 for the only rotor and the compact-type wind turbines respectively. The power coefficient increases with decreasing the frequency $f$.

Let discuss the dependence of the wind frequency on the wind turbine types. The power coefficients in the steady wind are smaller than ones of the oscillating winds of $f=0.033,0.05$ and $0.083 \mathrm{~Hz}$ for the only rotor. While, the case of $f=0.25 \mathrm{~Hz}$ is smaller than one of steady wind for $\lambda=1.6$ to 2.8 . It is indicated that the rotor do not respond the oscillating wind at $f=0.25 \mathrm{~Hz}$ for the tip range of speed ratio. On the other hand, the power coefficients of the compact-type wind-lens turbine for all frequencies are larger than one of steady flow. It is made clear that the compact wind-lens turbine work well for 


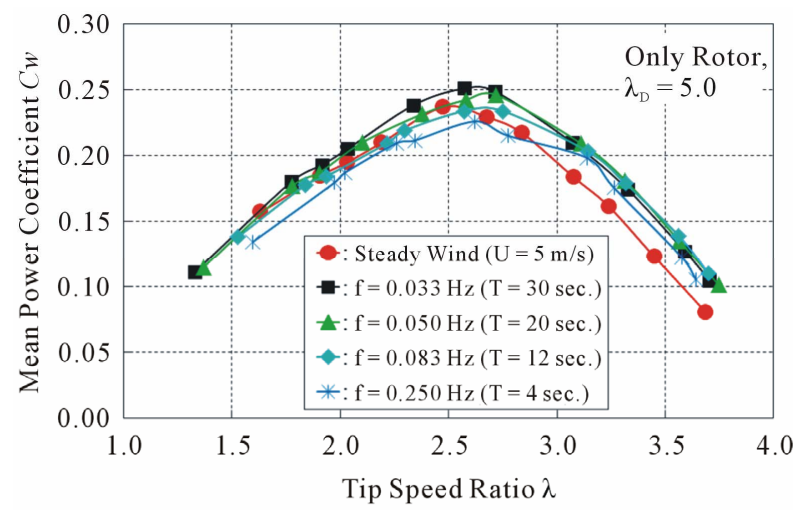

Figure 9. Effect of the wind frequencies $f$ on the time averaged power coefficients $C_{w}$ for the only rotor with $\bar{U}=5$ $\mathrm{m} / \mathrm{s}, \quad \tilde{\boldsymbol{u}}=1.0 \mathrm{~m} / \mathrm{s}(\tilde{\boldsymbol{u}} / \bar{U}=\mathbf{2 0} \%)$ and $\lambda_{D}=\mathbf{5 . 0}$.

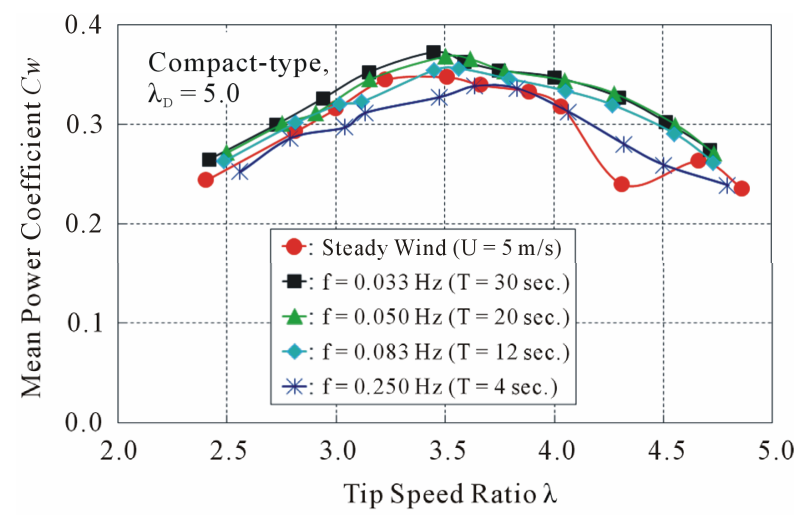

Figure 10. Effect of the wind frequencies $\boldsymbol{f}$ on the time averaged power coefficients $C_{w}$ for the compact-type with $\bar{U}$ $=5 \mathrm{~m} / \mathrm{s}, \quad \tilde{\boldsymbol{u}}=1.0 \mathrm{~m} / \mathrm{s}(\tilde{\boldsymbol{u}} / \bar{U}=20 \%)$ and $\lambda_{D}=5.0$.

the higher oscillating wind frequency. Therefore, the compact-type wind-lens turbine is suitable for the oscillating velocity wind than the only rotor one.

In order to make clear the dependence of the turbine type on performance in the oscillating flow, we define the increasing ratio $\varepsilon$ of the maximum power coefficient on the basis of steady wind as follow,

$$
\varepsilon=\frac{\tilde{C}_{w}-\bar{C}_{w}}{\bar{C}_{w}},
$$

where $\tilde{C}_{w}$ and $\bar{C}_{w}$ are maximum power coefficients in unsteady and steady winds respectively. Figure 11 presents the increasing ratio $\varepsilon$ for the only rotor and the compact-type with $\lambda_{D}=5.0$. As you see, the compacttype turbine is better than the only rotor.

\subsection{Numerical Estimation of the Power Coefficient of the Wind Turbines in Oscillating Flow}

Karasudani et al. suggest that the numerical estimation of

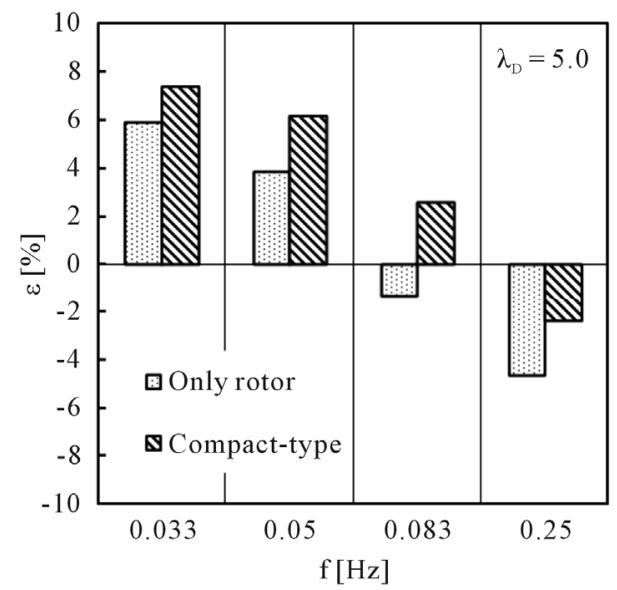

Figure 11. Increasing ratio of maximum power coefficient for oscillating wind on the basis of one for steady wind.

turbine performances for the oscillating wind [4]. Here it is assumed that the oscillating wind velocity $U(t)$ consists of the linear $u(t)$ he unsteady component $u(t)$ does not correlate to the unsteady power coefficient of a wind turbine. The time averaged power coefficient $C_{w}$ of the rotor is determined through the steady power coefficient $C_{w}^{A}$ as the following Equations (5) to (12),

$$
\begin{gathered}
C_{w} \cong C_{w}^{A}\left(1+3 \sigma^{2}\right), \\
C_{w}^{A}=\frac{1}{2 g\left(f / f_{r}\right) \Delta \lambda_{\infty}} \int_{\lambda_{-}}^{\lambda_{+}} C_{w}(\lambda) \mathrm{d} \lambda, \\
\lambda_{ \pm}=\lambda_{w} \pm g\left(f / f_{r}\right)+h\left(f / f_{r}\right) \varepsilon \lambda_{\infty}, \\
\Delta \lambda_{\infty}=\lambda_{w} \sqrt{2} \sigma, \\
\varepsilon \lambda_{\infty}=\lambda_{w} 2 \sigma^{2}, \\
\sigma^{2} \equiv \frac{1}{T} \int_{0}^{T}\left\{\frac{u(t)}{\bar{U}}\right\}^{2} \mathrm{~d} t=\frac{1}{2}\left(\frac{u(t)}{\bar{U}}\right)^{2}, \\
g\left(f / f_{r}\right)=1.04\left\{1-\exp \left(-7.49 \frac{f}{f_{r}}\right)\right\}, \\
h\left(f / f_{r}\right)=1.37\left\{1-\exp \left(-73.76 \frac{f}{f_{r}}\right)\right\} .
\end{gathered}
$$

Here it is mentioned that the approximated terms of $g\left(f / f_{r}\right)$ and $h\left(f / f_{r}\right)$ are independent of moment of inertia, rotor radius, power coefficient and control time.

The power coefficient in unsteady wind is $3 \sigma^{2}$ larger than one in the steady wind. The power coefficient ratio $\eta$ is defined to compare the experiment result $C_{w E}$ and numerical estimation $C_{w T}$,

$$
\eta=\frac{C_{w E}}{C_{w T}}=\frac{C_{w E}}{C_{w}^{A}\left(1+3 \sigma^{2}\right)} .
$$


Figure 12 summarizes $\eta$ for all cases. The only rotor and compact-type turbines are illustrated from left to light for each frequency, the same pattern of bar graph presents also the wind turbine type. As you see, the numerical estimation can predict the power coefficients in the oscillating flows to an accuracy of $94 \%$ to $102 \%$.

\section{Performance in Natural Fluctuating Velocity}

The natural fluctuating wind is generated by the active controlled multi-fan wind tunnel on the basis of Karman's power spectral equation as follows:

$$
\begin{gathered}
S_{u}(f)=4 I_{t}^{2} L_{v} \bar{U} \frac{1}{\left[1+70.8\left(f L_{v} / \bar{U}\right)^{2}\right]^{5 / 6}}, \\
I_{t}=\frac{\sigma}{\bar{U}} .
\end{gathered}
$$

Here, $I_{t}$ and $L_{v}$ mean turbulent intensity and vortex scale respectively. We examine the effect of the main factors of the natural wind on power coefficients of the only rotor and the compact-type wind turbines for mean wind velocity $5 \mathrm{~m} / \mathrm{s}$. Experimental condition and the effect of turbulent intensity on power coefficient are shown in Table 4 and Figure 13 respectively. According to experimental results, both $I_{t}$ and $L_{v}$ increase with increasing $C_{w}$ for both only rotor and compact-type wind turbines.

\section{Conclusions}

This paper presented wind performances of the only rotor turbine and flanged diffuser shroud which are so called "the compact-type wind-lens turbine" in steady and unsteady winds.

The flow structure around the wind lens turbine is made clear by CFD and PIV in steady wind. Also, it is shown that the compact wind-lens turbine works larger

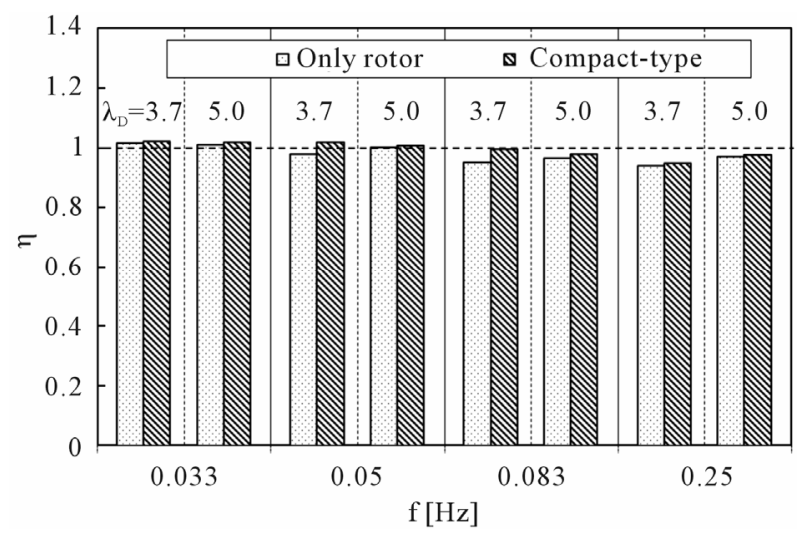

Figure 12. Comparisons of the experimental and numerical maximum power coefficients of the wind turbines.

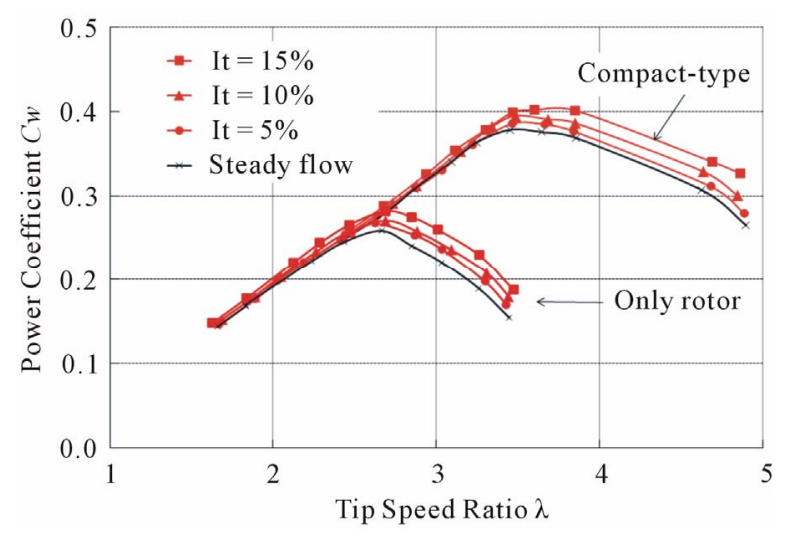

Figure 13. Effect of the turbulent intensity on power coefficient for the natural fluctuating wind of time average wind velocity $5 \mathrm{~m} / \mathrm{s}$ and vortex scale $3 \mathrm{~m}$.

Table 4. Natural fluctuating wind conditions at $\bar{U}=5 \mathrm{~m} / \mathrm{s}$.

\begin{tabular}{ccccc}
\hline Case 1 & $I_{t}$ & & $L_{v}$ \\
& $5 \%$ & $10 \%$ & $15 \%$ & $3 \mathrm{~m}$ \\
\hline \multirow{2}{*}{ Case 2} & $L_{v}$ & & $I_{t}$ \\
& $1 \mathrm{~m}$ & $3 \mathrm{~m}$ & $6 \mathrm{~m}$ & $10 \%$ \\
\hline
\end{tabular}

power generator than the only rotor wind turbine.

The performance of the wind turbines is investigated in the harmonic oscillating velocity wind of the upstream mean velocities $\bar{U}=5 \mathrm{~m} / \mathrm{s}$ with oscillating amplitude $\tilde{u}=1.0 \mathrm{~m} / \mathrm{s}$ at frequencies $f=0.033,0.05,0.083$ and $0.25 \mathrm{~Hz}$. The experimental and numerical results are presented to make clear the dependence of the wind velocity frequency on power coefficient of the wind turbines in sinusoidally oscillating velocity wind.

Consequently, the compact-type wind-lens turbine indicates the higher performance than an only rotor one in both steady and unsteady wind. In particular using the present design rotor, the wind-lens turbine is suitable for the oscillating velocity wind. The characteristic frequency proposed by Karasudani can be one of index to estimate turbine performance in the oscillating wind. In the experiment, the numerical estimation can predict the power coefficients within $94 \%$ to $102 \%$ accuracy in the harmonic oscillating wind.

Furthermore, performance dependence on turbulent intensity and vortex scale of natural fluctuating velocity wind is investigated. Both factors are increase with increasing power coefficient for the only rotor and compact-type wind lens turbines.

\section{Acknowledgements}

The work was supported in part by the Grant-in-Aid for Scientific Research through grant number 21560823 from the Ministry of Education, Science, and Culture of 
Japan, and the Collaborative Research Program of Research Institute for Applied Mechanics, Kyushu University. The authors gratefully acknowledge the support of Prof. Yuji Ohya, Prof Takashi Karasudani and Prof. Masato Furukawa of Kyushu University, to this investigation.

\section{REFERENCES}

[1] Y. Ohya and T. Karasudani, "A Shrouded Wind Turbine Generating High Output Power with Wind-Lens Technology," Journal of Energies, Vol. 3, No. 4, 2010, pp. 643649.

[2] K. Toshimitsu, K. Nishikawa, W. Haruki, S. Oono, M. Takao and Y. Ohya, "PIV Measurement of Flows around the Wind Turbines with a Flanged-Diffuser Shroud," Journal of Thermal Science, Vol. 17, No. 4, 2008, pp. 375380 .
[3] T. Karasudani, Y. Ohya and K. Watanabe, "On the Response of Small Wind Turbine to Changes of Wind Speed and Load," Journal of Japan Wind Energy Association, Vol. 31, No. 2, 2007, pp.120-123. (in Japanese)

[4] T. Karasudani, Y. Ohya and K. Watanabe, "On the Power of a Wind Turbine with Fixed-Pitch Angle Wings for Sinusoidally Changing Wind Speed," Journal of Japan Wind Energy Association, Vol. 32, No. 1, 2008, pp. 128132. (in Japanese)

[5] M. Okino, S. Iba, T. Karasudani, Y. Ohya and K. Watanabe, "Effect of Atmospheric Turbulence on Wind Turbine Performance," Proceedings of the 29th Symposium of Wind Energy Utilization, Tokyo, 2007, pp. 310-313. (in Japanese)

[6] Y. Sugita, M. Ueda and M. Furukawa, "Flow Structure around a Wind Turbine Shrouded by Compact-Type Brimmed Diffuser," Proceedings of JSME Fluid Engineering Conference 2005 (CD-ROM ISSN 1348-2882), Kanazawa, 2005, Paper No. 1819. (in Japanese) 